\title{
Equações preditivas e valores de normalidade para pressões respiratórias máximas na infância e adolescência
}

\author{
Predictive equations and normal values for maximal respiratory pressures in childhood and adolescence
}

\author{
Diana Amélia de Freitas ${ }^{1}$, Raíssa de Oliveira Borja ${ }^{2}$, Gardênia Maria H. Ferreira ${ }^{3}$, Patrícia Angélica de M. S. Nogueira ${ }^{4}$, Karla Morganna \\ P. P. de Mendonça ${ }^{3}$
}

\section{RESUMO}

Objetivo: Pesquisar equações preditivas e valores de normalidade para pressões respiratórias máximas disponíveis na literatura para a faixa etária compreendida entre a infância e a adolescência.

Fontes de dados: Estudos publicados em inglês e em português no período entre 1980 e 2009. As bases de dados eletrônicas Lilacs e Medline foram consultadas utilizando-se as palavras-chave "capacidade respiratória máxima”, "músculos respiratórios", "valores de referência”, "adolescente" e "criança”.

Síntese dos dados: Foram incluídos oito artigos na revisão, totalizando 1.463 crianças e adolescentes avaliados. A faixa etária da população estudada variou de sete a 18 anos. Geralmente o indivíduo é avaliado na posição sentada e com um clipe nasal. Os esforços máximos são realizados a partir do volume residual e da capacidade pulmonar total e sustentados por um a três segundos. Valores de normalidade e equações de predição foram propostos em oito e dois estudos, respectivamente. Nestes, demonstra-se incremento nas pressões respiratórias máximas desde a infância à adolescência e a ocorrência de maiores valores de pressão expiratória máxima quando comparados à pressão inspiratória máxima em crianças e adolescentes de ambos os sexos.

Conclusões: As pressões respiratórias máximas constituem um meio efetivo para avaliar a força muscular respiratória e diversos fatores contribuem para a grande variedade de equações preditivas e de valores de normalidade disponíveis. É preciso buscar um consenso para normatizar os métodos requeridos ao avaliar a força muscular respiratória em crianças e adolescentes.
Palavras-chave: força muscular; músculos respiratórios; valores de referência; adolescente; criança.

\section{ABSTRACT}

Objective: To investigate predictive equations and normal values for maximal respiratory pressures available in the literature for children and adolescents.

Data sources: Studies in English and Portuguese published from 1980 to 2009. Lilacs and Medline databases were consulted using the key-words "reference values", "respiratory muscles", "maximal respiratory capacity", "adolescent" and "child".

Data synthesis: Eight articles with a total of 1,463 adolescents and children were included in the review. The age of the studied population ranged from seven to 18 years old. The evaluations were usually performed with the patients in the sitting position with a nose clip. The variables were measured from residual volume and from total lung capacity and they were sustained for 1-3 seconds. Normal values and predictive equations were proposed in eight and two studies, respectively. These studies showed an increase in maximal respiratory pressures from childhood to adolescence and higher values of maximal expiratory pressure compared with maximal inspiratory pressure in children and adolescents of both genders.

Conclusions: Maximal respiratory pressures are an effective way to assess respiratory muscle strength and several factors contribute to the wide variety of predictive equations and normal values available. Consensus to standardize the methods
Instituição: Departamento de Fisioterapia da Universidade Federal do Rio Grande do Norte (UFRN), Natal, RN, Brasil

'Graduada em Fisioterapia pela UFRN, Natal, RN, Brasil

${ }^{2}$ Mestranda em Fisioterapia pelo Programa de Pós-Graduação em Fisioterapia da UFRN; Fisioterapeuta do Hospital Estadual Dr. Ruy Pereira dos Santos, Natal, RN, Brasil

${ }^{3}$ Doutora em Ciências da Saúde pela UFRN; Professora Adjunta do Departamento de Fisioterapia da UFRN, Natal, RN, Brasil

${ }^{4}$ Doutora em Ciências pelo Programa de Cardiologia da Universidade Federal de São Paulo (Unifesp); Professora Adjunta do Departamento de Fisioterapia da UFRN, Natal, RN, Brasil
Endereço para correspondência:

Karla Morganna P. P. de Mendonça

Avenida Ayrton Senna, 880 Casa 82 - Condomínio Bosque das Palmeiras/

Cidade dos Bosques - Nova Parnamirim

CEP 59151-600 - Parnamirim/RN

E-mail: kmorganna@ufrnet.br

Conflito de interesse: nada a declarar

Recebido em: 23/7/2010

Aprovado em: 26/1/2011 
required to assess respiratory muscle strength in children and adolescents is needed.

Key-words: muscle strength; respiratory muscles; reference values; adolescent; child.

\section{Introdução}

Os músculos respiratórios são responsáveis pelo adequado funcionamento do sistema respiratório. A mensuração da força muscular respiratória permite identificar precocemente quadros de fraqueza muscular respiratória e de insuficiência respiratória ${ }^{(1)}$, proporciona maior segurança no tratamento de pacientes ${ }^{(2)}$, indica a gravidade, as consequências funcionais e o progresso de diversas disfunções pulmonares e neuromusculares ${ }^{(3)}$, além de contribuir para um melhor acompanhamento do processo de desmame da ventilação mecânica ${ }^{(4)}$.

Existem diversos procedimentos invasivos ou não para avaliar a força muscular respiratória. As pressões inspiratória e expiratória máximas (PImáx e PEmáx, respectivamente) constituem um método de avaliação não invasiva frequente e clinicamente útil. Trata-se da mensuração da pressão intraoral durante a realização de um esforço inspiratório ou expiratório máximo, contra a via aérea ocluída. A PImáx avalia a força dos músculos inspiratórios, enquanto a PEmáx, a força dos músculos expiratórios ${ }^{(5)}$.

Ao longo dos anos, muitos estudos, com diferentes técnicas, foram realizados para obter valores de normalidade e equações de referência de pressões respiratórias máximas para populações saudáveis de várias faixas etárias e de diversos grupos étnicos ${ }^{(6-10)}$.

Esse estudo se propôs a reunir os achados referentes aos valores de normalidade e às equações de predição para pressões respiratórias máximas para crianças e adolescentes saudáveis, a fim de facilitar a escolha do método e dos parâmetros mais adequados para uma correta avaliação dos pacientes, bem como a adequada interpretação dos valores obtidos.

\section{Métodos}

A pesquisa dos artigos científicos abrangeu todos os estudos publicados em português e inglês, no período entre 1980 e 2009, nas bases de dados eletrônicas Lilacs e Medline, que disponibilizaram valores de referência e/ou equações preditivas para pressões respiratórias máximas de crianças e adolescentes saudáveis. Foram utilizadas as seguintes palavras chaves: "capacidade respiratória máxima” ("maximal respiratory capacity"), "músculos respiratórios" ("respiratory muscles"), "valores de referência" ("reference values"), "adolescente" ("adolescent") e "criança" ("child"). Além disso, também foi feita consulta direta aos sites de revistas da área, de acordo com as listas de referências dos artigos pré-selecionados.

Os estudos incluídos nesta revisão foram minuciosamente avaliados para que seus métodos pudessem ser detalhados. Os aspectos avaliados e, posteriormente, descritos em tabelas foram: 1) A faixa etária da amostra estudada; 2) O tamanho da amostra; 3) A postura adotada pelo sujeito avaliado durante a execução das pressões respiratórias máximas; 4) A utilização ou não de clipe nasal; 5) Os volumes e as capacidades a partir das quais foram avaliadas a PImáx e a PEmáx; 6) O número de repetições das manobras; 7) O tempo de sustentação do esforço; 8) As variáveis independentes avaliadas; 9) Os valores de normalidade de PImáx e PEmáx propostos para ambos os sexos; e 10) As equações preditivas de PImáx e PEmáx, também para ambos os sexos.

\section{Resultados}

$\mathrm{Na}$ pesquisa realizada nas bases de dados eletrônicas, foram encontrados nove artigos, dos quais um foi excluído por não descrever claramente o método empregado. Dessa forma, foram incluídos oito artigos na revisão, todos envolvendo a avaliação da musculatura respiratória por meio das pressões respiratórias máximas em crianças e adolescentes saudáveis na faixa etária entre sete e 18 anos. Analisaram-se os dados obtidos nos oito artigos, totalizando uma amostra de 1.463 crianças e adolescentes; as amostram variaram de 40 a 392 participantes nos estudos incluídos. Não foram encontrados artigos com valores de normalidade para pressões respiratórias máximas em crianças e adolescentes brasileiros.

A Tabela 1 mostra os oito estudos incluídos na revisão. É possível observar separadamente as características da amostra (número, distribuição entre os sexos e faixa etária), as técnicas utilizadas no protocolo de avaliação (posição de avaliação, utilização ou não de clipe nasal, tempo de sustentação do esforço máximo, volumes e capacidades pulmonares nos quais os esforços máximos iniciaram e número de repetição das manobras) e as variáveis independentes relacionadas. Nas tabelas 2 e 3 estão expostos os valores de normalidade e as equações preditivas para PImáx e PEmáx propostas nos oito estudos revisados para os sexos feminino e masculino, respectivamente. 


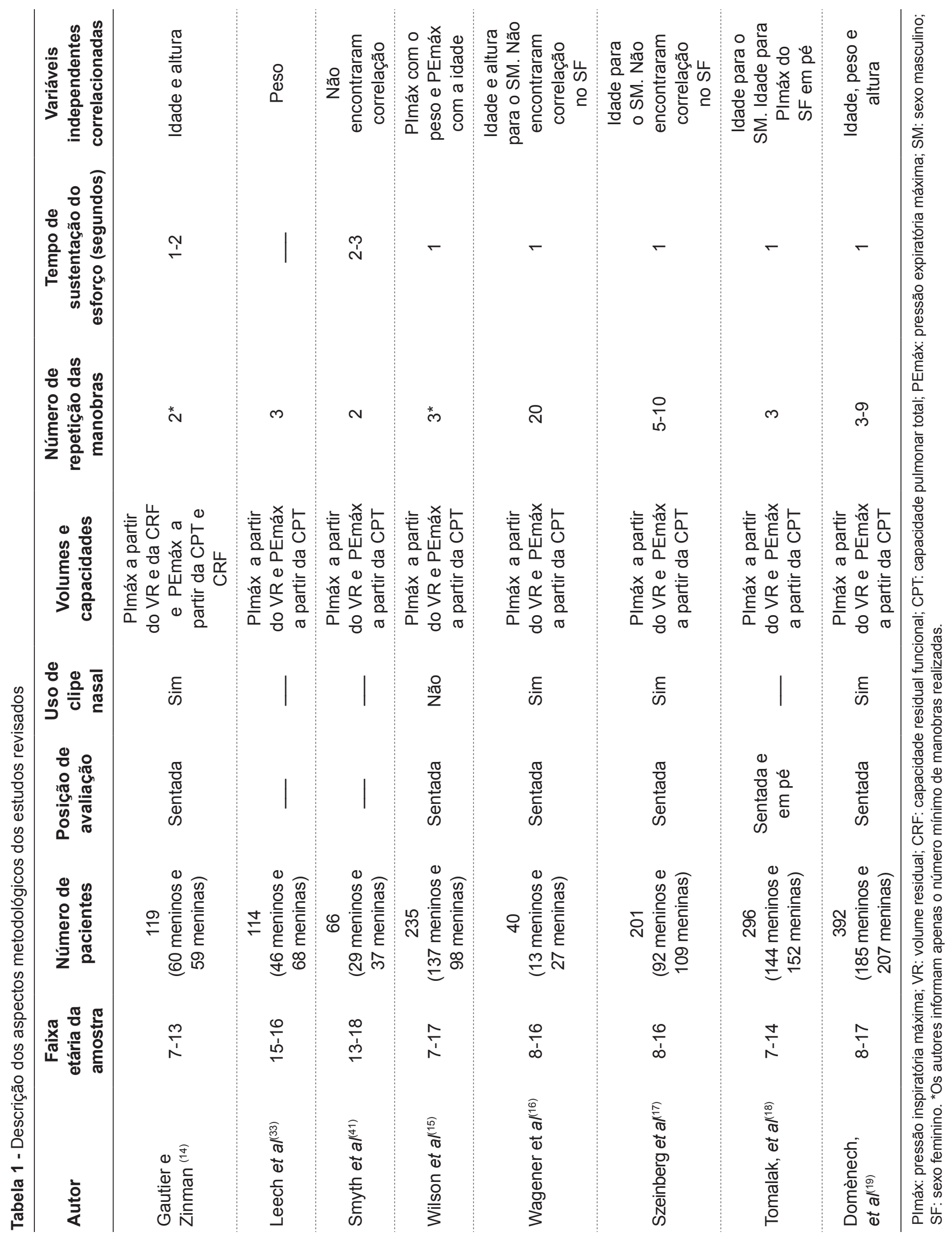


Tabela 2 - Valores de normalidade e/ou equações preditivas para pressão inspiratória máxima e pressão expiratória máxima para o sexo feminino disponibilizados pelos estudos revisados

\begin{tabular}{|c|c|c|c|c|}
\hline Autor & $\begin{array}{c}\text { Valores de } \\
\text { normalidade para } \\
\text { PImáx }_{\mathrm{VR}}\left(\mathrm{cmH}_{2} \mathrm{O}\right)^{\#}\end{array}$ & $\begin{array}{c}\text { Valores de } \\
\text { normalidade para } \\
\text { PEmáx }_{\mathrm{CPT}}\left(\mathrm{cmH}_{2} \mathrm{O}\right)^{\#}\end{array}$ & $\begin{array}{c}\text { Equações preditivas } \\
\text { para PImáx }\end{array}$ & $\begin{array}{l}\text { Equações preditivas } \\
\text { para PEmáx }\end{array}$ \\
\hline $\begin{array}{l}\text { Gautier e } \\
\text { Zinman }^{(14)}\end{array}$ & $\begin{array}{c}\text { 7-8: }-71(29) \\
\text { 9-10: }-86(25) \\
11-13:-108(29)\end{array}$ & $\begin{array}{c}\text { 7-8: } 74(25) \\
\text { 9-10: } 108(39) \\
\text { 11-13: } 126(32)\end{array}$ & - & - \\
\hline Leech et $a /(33)$ & $-85(28)$ & $95(29)$ & - & - \\
\hline Smyth et $a /(41)$ & $-76(25)$ & $86(22)$ & - & - \\
\hline Wilson et $a^{/(15)}$ & $-63(21)$ & $80(21)$ & $40+(0,57 \times$ peso $)$ & $24+(4,8 \times$ idade $)$ \\
\hline Wagener et $a^{/(16)}$ & $-113(24)^{*}$ & $142(36)^{*}$ & - & - \\
\hline Szeinberg et a/(17) & $\begin{array}{l}\text { 8-10: }-104(20) \\
\text { 11-13: }-112(20) \\
\text { 14-16: }-109(21)\end{array}$ & $\begin{array}{l}\text { 8-10: } 129(29) \\
\text { 11-13: } 138(31) \\
\text { 14-16: } 135(29)\end{array}$ & - & - \\
\hline Tomalak et a/(18) & $\begin{array}{l}\text { Sentada: }-66(20)^{*} \\
\text { Em pé: }-67(20)^{*}\end{array}$ & $\begin{array}{l}\text { Sentada: } 70(18)^{*} \\
\text { Em pé: } 72(18)^{*}\end{array}$ & - & - \\
\hline Domènech et a/(19) & $\begin{array}{l}\text { 8-10: - } 68(24) \\
\text { 11-14: }-89(27) \\
\text { 15-17: }-97(24)\end{array}$ & $\begin{array}{c}\text { 8-10: } 82(29) \\
\text { 11-14: } 115(33) \\
\text { 15-17: } 133(35)\end{array}$ & $\begin{array}{l}-33,854-(1,814 \times \text { idade }) \\
-(0,004 \times \text { altura } x \text { peso })\end{array}$ & $17,066+(7,22 \times$ idade $)$ \\
\hline
\end{tabular}

\#Os valores de normalidade são expressos em média e desvio padrão e podem apresentar subdivisões por faixa etária ou posição de avaliação; *valores originalmente expressos em kilopascal $(\mathrm{KPa})$, convertidos para centímetros de água $\left(\mathrm{cmH}_{2} \mathrm{O}\right),\left(1 \mathrm{KPa}=10,2 \mathrm{cmH} \mathrm{H}_{2} \mathrm{O}\right)$. PImáx: pressão inspiratória máxima; VR: volume residual; PEmáx: pressão expiratória máxima; CPT: capacidade pulmonar total.

Tabela 3 - Valores de normalidade e/ou equações preditivas para pressão inspiratória máxima e pressão expiratória máxima para o sexo masculino disponibilizados pelos estudos revisados

\begin{tabular}{|c|c|c|c|c|}
\hline Autor & $\begin{array}{c}\text { Valores de } \\
\text { normalidade para } \\
\text { PImáx }_{\mathrm{VR}}\left(\mathrm{cmH}_{2} \mathrm{O}\right)^{\#}\end{array}$ & $\begin{array}{c}\text { Valores de } \\
\text { normalidade para } \\
\text { PEmáx }_{\mathrm{CPT}}\left(\mathrm{cmH}_{2} \mathrm{O}\right)^{\#}\end{array}$ & $\begin{array}{c}\text { Equações preditivas } \\
\text { para PImáx }\end{array}$ & $\begin{array}{c}\text { Equações preditivas } \\
\text { para PEmáx }\end{array}$ \\
\hline Gautier e Zinman ${ }^{(14)}$ & $\begin{array}{c}\text { 7-8: }-77(24) \\
\text { 9-10: }-105(27) \\
\text { 11-13: }-114(27)\end{array}$ & $\begin{array}{c}\text { 7-8: } 99(23) \\
\text { 9-10: } 123(27) \\
\text { 11-13: } 161(37)\end{array}$ & - & - \\
\hline Leech et $a /^{(33)}$ & $-111(34)$ & $131(30)$ & - & - \\
\hline Smyth et a/(41) & $-107(26)$ & $114(35)$ & - & - \\
\hline Wilson et $a^{/(15)}$ & $-75(23)$ & $96(23)$ & $44,5+(0,75 \times$ peso $)$ & $35+(5,5 \times$ idade $)$ \\
\hline Wagener et $a^{/(16)}$ & $-113(24)^{*}$ & $142(36)^{\star}$ & - & - \\
\hline Szeinberg et a/(17) & $\begin{array}{c}\text { 8-10: }-116(26) \\
\text { 11-13: }-130(16) \\
\text { 14-16: }-126(22))\end{array}$ & $\begin{array}{l}\text { 8-10: } 142(25) \\
\text { 11-13: } 176(24) \\
\text { 14-16: } 166(44)\end{array}$ & - & - \\
\hline Tomalak et a/(18) & $\begin{array}{l}\text { Sentados: }-84(27)^{*} \\
\text { Em pé: }-83(27)^{*}\end{array}$ & $\begin{array}{l}\text { Sentados: } 81(23)^{*} \\
\text { Em pé: } 80(23)^{*}\end{array}$ & - & - \\
\hline Domènech et $a^{(19)}$ & $\begin{array}{c}\text { 8-10: -79 (31) } \\
\text { 11-14: }-111(31) \\
\text { 15-17: }-129(24))\end{array}$ & $\begin{array}{c}\text { 8-10: } 95(34) \\
\text { 11-14: } 147(34) \\
\text { 15-17: } 180(43)\end{array}$ & $\begin{array}{l}-27,020-(4,132 \times \text { idade }) \\
-(0,003 \times \text { altura } \times \text { peso })\end{array}$ & $\begin{array}{r}7,619+(7,806 \times \text { idade }) \\
+(0,004 \times \text { altura } \times \text { peso })\end{array}$ \\
\hline
\end{tabular}

Os valores de normalidade são expressos em média e desvio padrão e poderão apresentar subdivisões por faixa etária ou posição de avaliação; *valores originalmente expressos em kilopascal (KPa), convertidos para centímetros de água $\left(\mathrm{cmH}_{2} \mathrm{O}\right),\left(1 \mathrm{KPa}=10,2 \mathrm{cmH} \mathrm{H}_{2} \mathrm{O}\right)$. PImáx: pressão inspiratória máxima; VR: volume residual; PEmáx: pressão expiratória máxima; CPT: capacidade pulmonar total. 


\section{Discussão}

A avaliação das pressões respiratórias máximas é um teste de esforço dependente; dessa forma, torna-se difícil realizá-la em crianças muito jovens ${ }^{(11)}$. A idade mínima das crianças incluídas nos estudos revisados foi de sete anos. Em um artigo sobre testes de função pulmonar em crianças préescolares publicado em 2007, a American Thoracic Society e a European Respiratory Society ${ }^{(12)}$ analisaram a dificuldade em se avaliar a função pulmonar de crianças na faixa etária entre dois e seis anos de idade, porém afirmam que sua realização é de grande importância, tanto por razões clínicas quanto pelo crescimento e desenvolvimento do sistema respiratório que ocorre nesse período. Os autores ressaltaram ainda que os valores de normalidade para testes de função pulmonar encontrados para crianças mais velhas não podem ser utilizados para as menores de seis anos de idade. Embora equações preditivas para alguns testes de função pulmonar em crianças pré-escolares estejam disponíveis na literatura ${ }^{(13)}$, permanece a lacuna no que se refere a valores de normalidade e a equações preditivas para avaliar a força muscular respiratória de crianças nessa faixa etária.

$\mathrm{Na}$ presente revisão, observou-se uma maior frequência de avaliações realizadas com os participantes na posição sentada ${ }^{(14-19)}$. No entanto, Tomalak et $a l^{(18)}$, ao estudarem os participantes na posição sentada e em pé, não evidenciaram diferenças para os valores de PImáx e PEmáx nessas duas posições. Achados semelhantes foram encontrados por Fiz et al ${ }^{(20)}$, ao compararem os valores encontrados para as pressões respiratórias máximas nessas mesmas posturas.

Os estudos de Wilson et a ${ }^{(15)}$ e de Tomalak et $a l^{(18)}$ forneceram os menores valores de normalidade dentre os artigos revisados. Quanto aos achados de Wilson et al ${ }^{(15)}$, os valores obtidos podem ser atribuídos à não utilização de clipe nasal, o que favorece o escape aéreo durante as medidas. Tomalak et a ${ }^{(18)}$ não citaram, na descrição dos métodos, a aplicação ou não de clipe nasal. No entanto, a American Thoracic Society e a European Respiratory Society ${ }^{(21)}$, em artigo publicado em 2002, afirmaram que o uso de clipe nasal não é necessário.

Todos os estudos revisados iniciaram a PImáx e a PEmáx a partir do volume residual e da capacidade pulmonar total, respectivamente. Destes, apenas um também avaliou os esforços máximos a partir da capacidade residual funcional ${ }^{(14)}$. A mensuração das pressões respiratórias máximas é realizada por convenção a partir do volume residual e da capacidade pulmonar total ${ }^{(21)}$. No entanto, a mensuração da PImáx a partir do volume residual tem a desvantagem de o valor obtido sofrer interferência da força de recolhimento elástico do sistema respiratório ${ }^{(22)}$.

O tempo de sustentação dos esforços máximos nos artigos revisados variou de um a três segundos. Segundo Souza ${ }^{(23)}$, o esforço máximo deve ser mantido por um breve período de tempo e essa duração pode variar de um a três segundos, dependendo do autor. Windisch $e t$ al ${ }^{(24)}$ avaliaram a PImáx em uma população saudável de dez a 90 anos, registrando os valores de pico e os de platô. Esses autores encontraram valores para pressão de pico significativamente maiores do que os de platô, no entanto, afirmaram que não há desvantagem em se utilizar a pressão de pico em vez da pressão platô. Wijkstra et al ${ }^{(25)}$, ao compararem a pressão de pico em uma população saudável e em pacientes com doença pulmonar obstrutiva crônica (DPOC), concluíram que a pressão de pico é um recurso válido e não invasivo para se avaliar a força muscular respiratória.

O número de manobras variou entre dois e 20, corroborando, este último, com o efeito de aprendizado ${ }^{(26)}$. De acordo com Fiz et $a l^{(27)}$, em pacientes com limitação ao fluxo aéreo, um mínimo de nove manobras é necessário para obter um valor máximo para PImáx. Em 1997, Wen et al ${ }^{(28)}$ afirmaram que quanto maior o número de manobras, mais precisa a avaliação da fraqueza muscular. Em 2008, Alexandre $e t$ a $l^{(1)}$ preconizaram a realização de três a cinco manobras, para que se possam obter três manobras aceitáveis, nas quais não haja vazamento e, dessas três, deve haver duas reprodutíveis (diferença menor que $5 \%$ entre elas).

A análise dos valores de normalidade fornecidos pelos estudos revisados mostrou que as pressões respiratórias máximas são maiores em meninos do que em meninas e que os valores para PEmáx superam os para PImáx em ambos os sexos. Em um estudo para obter valores de normalidade para pressão inspiratória nasal em crianças e adolescentes, Stefanutti e Fitting ${ }^{(29)}$ avaliaram as PEmáx e PImáx e observaram maiores pressões respiratórias máximas nos meninos. Esse estudo também apresentou valores mais elevados para PEmáx em ambos os sexos. Santiago et al ${ }^{(30)}$, ao avaliarem a força muscular respiratória em dois grupos de crianças brasileiras, sendo um formado por crianças com sobrepeso e outro por crianças eutróficas, obtiveram maiores valores para PEmáx do que para PImáx no grupo de eutróficos. Esse último estudo, no entanto, não separou os valores obtidos por sexo.

As pressões respiratórias máximas aumentam com a idade. Prioux $e t$ a $l^{(31)}$ e Matecki $e t a l^{(32)}$ avaliaram durante três anos as pressões respiratórias máximas de pré-adolescentes e adolescentes franceses do sexo masculino, divididos em três grupos 
(pré-puberdade, puberdade e pós-puberdade). O primeiro estudo observou que as pressões respiratórias máximas tendem a aumentar entre os 11 e 17 anos. O estudo de Matecki et al ${ }^{(32)}$, por sua vez, indicou aumento significativo da PImáx entre 11 e 17 anos e da PEmáx entre 11 e 15 anos.

A idade foi a variável independente que mais se relacionou aos valores obtidos pelos estudos revisados ${ }^{(14-19)}$, seguida pelo peso $^{(15,19,33)}$ e altura ${ }^{(14,16,19)}$. Ao se avaliar a pressão inspiratória nasal em crianças e comparar os valores obtidos com os de PImáx, Rafferty et al ${ }^{(34)}$ encontraram correlação entre os valores de PImáx com idade, peso e altura. Trata-se de achado frequente, mesmo em estudos com populações distintas e em diversas faixas etárias ${ }^{(35)}$. Neder $e^{2} a^{(36)}$, ao avaliarem as pressões respiratórias máximas em uma população brasileira saudável na faixa etária entre 20 e 80 anos, observaram que a idade se correlacionou às pressões respiratórias máximas em ambos os sexos. O peso correlacionou-se apenas com a PImáx no sexo masculino.

Apenas dois estudos forneceram equações preditivas para a população estudada ${ }^{(15,19)}$ (Tabelas 2 e 3). Wilson et al ${ }^{(15)}$ incluíram as variáveis "peso" e "idade" nas equações preditivas para PImáx e PEmáx, respectivamente, para ambos os sexos. Domènech et al ${ }^{(19)}$ incluíram as variáveis "peso", "altura" e "idade" nas equações preditivas da PImáx para meninos e meninas e da PEmáx apenas para meninos. A equação proposta para a PEmáx em meninas incluiu apenas a variável "idade", porém os autores afirmaram que tal equação não pode ser considerada como preditiva para meninas.

Wilson et al ${ }^{(15)}$ também calcularam equações preditivas para adultos, nas quais incluíram a idade para PImáx e PEmáx nos homens e altura para PImáx e PEmáx em mulheres. Em estudo recente realizado no Brasil com indivíduos sedentários saudáveis entre 20 e 89 anos, Simões $e t$ a ${ }^{(37)}$ incluíram a variável "idade" nas equações preditivas para PImáx e PEmáx em homens e "idade" e "peso" nas equações para PImáx e PEmáx em mulheres.

Durante consulta às bases de dados não foram localizados estudos com valores de normalidade e equações preditivas para crianças e adolescentes brasileiros. Sabese que os valores obtidos para as pressões respiratórias máximas podem variar de acordo com os diferentes grupos étnicos ${ }^{(38)}$. Dessa forma, os valores de normalidade e as equações preditivas para a população brasileira podem diferir daqueles propostos para outros países ${ }^{(39)}$. Parreira et $a^{(40)}$ compararam os valores encontrados para pressões respiratórias máximas de uma amostra da população brasileira saudável do Estado de Minas Gerais com aqueles preditos pela equação de Neder et al ${ }^{(36)}$, concluindo que as equações propostas por Neder et al não foram capazes de prever os valores obtidos para as pressões respiratórias máximas da população avaliada, o que indica que as equações preditivas podem sofrer variações entre indivíduos de um mesmo país.

A presente revisão permitiu evidenciar que a avaliação da força muscular respiratória por meio das pressões respiratórias estáticas máximas constitui ainda um tema pouco abordado em crianças e adolescentes. Tal limitação é ainda mais expressiva no Brasil, pois ainda inexistem publicações que disponibilizem parâmetros de normalidade para essa faixa etária.

\section{Considerações finais}

As pressões respiratórias máximas constituem um meio efetivo para avaliar a força muscular respiratória. No entanto, as diferentes técnicas e os tipos de interfaces e aparelhos utilizados, bem como o nível de cooperação do sujeito avaliado, podem gerar diferenças nos valores encontrados.

Os estudos analisados mostraram que as pressões respiratórias máximas tendem a aumentar da infância à adolescência e que os valores de PEmáx são superiores aos encontrados para PImáx tanto em crianças quanto em adolescentes e em ambos os sexos. Foi possível também observar que a postura adotada durante a realização das manobras não interfere nas medidas obtidas e que a idade parece ser a variável que mais se correlaciona às pressões respiratórias máximas.

A avaliação das pressões respiratórias máximas é geralmente realizada com o indivíduo na posição sentada com um clipe nasal. A PImáx é comumente mensurada a partir do volume residual e a PEmáx, a partir da capacidade pulmonar total; o tempo de sustentação do esforço pode variar de um a três segundos. Recomenda-se a realização de três a cinco manobras para obter duas manobras aceitáveis, nas quais não haja escape de ar.

Este estudo de revisão permitiu disponibilizar equações preditivas e valores de normalidade para pressões respiratórias máximas recomendadas para crianças e adolescentes. A mesma pode ser considerada, ainda, uma ferramenta útil na busca de um consenso para normatizar os métodos requeridos ao avaliar a força muscular respiratória nessa faixa etária. 


\section{Referências bibliográficas}

1. Alexandre BL, Araújo SG, Machado MG. Pressões respiratórias máximas. In: Machado MG. Bases da fisioterapia respiratória: terapia intensiva e reabilitação. Rio de Janeiro: Guanabara Koogan; 2008. p. 111-24.

2. Kantor Junior $O$. Testes de função pulmonar em crianças e adolescentes. J Pediatr (Rio J) 1997;73:145-50.

3. Fiore Junior JF, Paisani DM, Franceschini J, Chiavegato LD, Faresin SM. Pressões respiratórias máximas e capacidade vital: comparação entre avaliações através de bocal e de máscara facial. J Bras Pneumol 2004;30:515-20.

4. Guimarães FS, Alves FF, Constantino SS, Dias CM, Menezes SL. Avaliação da pressão inspiratória máxima em pacientes críticos não-cooperativos: comparação entre dois métodos. Rev Bras Fisioter 2007;11:233-8.

5. Vasconcellos JA, Britto RR, Lopes RB. Avaliação da musculatura respiratória. In: Brittom AR, Brant TC, Parreira VF. Recursos manuais e instrumentais em fisioterapia respiratória. Barueri: Manole; 2009. p. 11-22.

6. Cook CD, Mead J, Orzalesi MM. Static volume-pressure characteristics of the respiratory system during maximal efforts. J Appl Physiol 1964;19:1016-22.

7. Black LF, Hyatt RE. Maximal respiratory pressures: normal values and relationship to age and sex. Am Rev Respir Dis 1969;99:696-702.

8. Camelo Junior JS, Terra-Filho J, Manço JC. Pressões respiratórias máximas em adultos normais. J Pneumol 1985;11:181-4.

9. Enright PL, Adams AB, Boyle PJ, Sherrill DL. Spirometry and maximal respiratory pressure references from healthy Minnesota 65- to 85-year-old women and men. Chest 1995;108:663-9.

10. Johan A, Chan CC, Chia HP, Chan OY, Wang YT. Maximal respiratory pressures in adult Chinese, Malays and Indians. Eur Respir J 1997;10:2825-8.

11. Fauroux B, Aubertin G. Measurement of maximal pressures and the sniff manoeuvre in children. Paediatr Respir Rev 2007;8:90-3.

12. Beydon N, Davis SD, Lombardi E, Allen JL, Arets HG, Aurora P et al. An official american thoracic society/european respiratory society statement: pulmonary function testing in preschool children. Am J Respir Crit Care Med 2007;175:1304-45.

13. Stanojevic S, Wade A, Lum S, Stocks J. Reference equations for pulmonary function tests in preschool children: a review. Pediatr Pulmonol 2007; $42: 962-72$.

14. Gaultier C, Zinman R. Maximal static pressures in healthy children. Respir Physiol 1983;51:45-61.

15. Wilson SH, Cooke NT, Edwards RH, Spiro SG. Predicted normal values for maximal respiratory pressures in Caucasian adults and children. Thorax 1984;39:535-8.

16. Wagener JS, Hibbert ME, Landau LI. Maximal respiratory pressures in children. Am Rev Respir Dis 1984;129:873-5.

17. Szeinberg A, Marcotte JE, Roizin H, Mindorff C, England S, Tabachnik E et al. Normal values of maximal inspiratory and expiratory pressures with a portable apparatus in children, adolescents and young adults. Pediatr Pulmonol 1987;3:255-8.

18. Tomalak W, Pogorzelski A, Prusak J. Normal values for maximal static inspiratory and expiratory pressures in healthy children. Pediatr Pulmonol 2002;34:42-6.

19. Domènech-Clar R, López-Andreu JA, Compte-Torrero L, De Diego-Damiá A, Macián-Gisbert V, Perpiñá-Tordera M et al. Maximal static respiratory pressures in children and adolescents. Pediatr Pulmonol 2003;35:126-32.

20. Fiz JA, Texidó A, Izquierdo J, Ruiz J, Roig J, Morera J. Postural variation of the maximum inspiratory and expiratory pressures in normal subjects. Chest 1990;97:313-4.

21. American Thoracic Society/European Respiratory Society. ATS/ERS statement on respiratory muscle testing. Am J Respir Crit Care Med 2002;166:518-624.

22. Polkey MI, Green M, Moxham J. Measurement of respiratory muscle strength. Thorax 1995; 50:1131-5.

23. Souza RB. Pressões respiratórias estáticas máximas. J Pneumol 2002;28 (Suppl 3):S155-65.

24. Windisch W, Hennings E, Sorichter S, Hamm H, Criée CP. Peak or plateau maximal inspiratory mouth pressure: which is best? Eur Respir J 2004;23:708-13.

25. Wijkstra PJ, van der Mark TW, Boezen M, van Altena R, Postma DS, Koeter $\mathrm{GH}$. Peak inspiratory mouth pressure in healthy subjects and in patients with COPD. Chest 1995;107:652-6.

26. Larson JL, Covey MK, Vitalo CA, Alex CG, Patel M, Kim MJ. Maximal inspiratory pressure. Learning effect and test-retest reliability in patients with chronic obstructive pulmonary disease. Chest 1993;104:448-53.

27. Fiz JA, Montserrat JM, Picado C, Plaza V, Agusti-Vidal A. How many maneuvers should be done to measure maximal inspiratory mouth pressure in patients with chronic airflow obstruction? Thorax 1989;44:419-21.

28. Wen AS, Woo MS, Keens TG. How many maneuvers are required to measure maximal inspiratory pressure accurately. Chest 1997;111:802-7.

29. Stefanutti D, Fitting JW. Sniff nasal inspiratory pressure. Reference values in Caucasian children. Am J Respir Crit Care Med 1999;159:107-11.

30. Santiago SQ, Silva ML, Davidson J, Aristóteles LR. Avaliação da força muscular respiratória em crianças e adolescentes com sobrepeso/obesos. Rev Paul Pediatr 2008;26:146-50.

31. Prioux J, Ramonatxo M, Mercier J, Granier P, Mercier B, Prefaut C. Changes in maximal exercise ventilation and breathing pattern in boys during growth: a mixed cross-sectional longitudinal study. Acta Physiol Scand 1997;161:447-58.

32. Matecki S, Prioux J, Jaber S, Hayot M, Prefaut C, Ramonatxo M. Respiratory pressures in boys from 11-17 years old: a semilongitudinal study. Pediatr Pulmonol 2003;35:368-74.

33. Leech JA, Ghezzo H, Stevens D, Becklake MR. Respiratory pressures and function in young adults. Am Rev Respir Dis 1983;128:17-23.

34. Rafferty GF, Leech S, Knight L, Moxham J, Greenough A. Sniff nasal inspiratory pressure in children. Pediatr Pulmonol 2000;29:468-75.

35. Harik-Khan RI, Wise RA, Fozard JL. Determinants of maximal inspiratory pressure. The Baltimore longitudinal study of aging. Am J Respir Crit Care Med 1998;158:1459-64.

36. Neder JA, Andreoni S, Lerario MC, Nery LE. Reference values for lung function tests. II. maximal respiratory pressures and voluntary ventilation. Braz J Med Biol Res 1999;32:719-27.

37. Simões RP, Deus AP, Auad MA, Dionísio J, Mazzonetto M, Borghi-SilvaA. Pressões respiratórias máximas em indivíduos saudáveis sedentários de 20 a 89 anos da região central do Estado de São Paulo. Rev Bras Fisioter 2010;14:60-7.

38. Evans JA, Whitelaw WA. The assessment of maximal respiratory mouth pressures in adults. Respir Care 2009;54:1348-59.

39. Leal AH, Hamasaki TA, Jamami M, Di Lorenzo VA, Pessoa BV. Comparação entre valores de força muscular respiratória medidos e previstos por diferentes equações. Fisioter Pesqui 2007;14:25-30.

40. Parreira VF, França DC, Zampa CC, Fonseca MM, Tomich GM, Britto RR. Pressões respiratórias máximas: valores encontrados e preditos em indivíduos saudáveis. Rev Bras Fisioter 2007;11:361-8. 